\title{
Spatial Frequency Domain Technique: An Approach for Analyzing the Scanning Laser Acoustic Microscope Interferogram Images
}

\author{
KALERVO M. U. TERVOLA AND WILLIAM D. O'BRIEN, JR., SENIOR MEMBER, IEEE
}

\begin{abstract}
An acoustic interferogram of equal phase wavefronts is produced with the scanning laser acoustic microscope. The interferogram contains information about the ultrasonic velocity distribution of the specimen under investigation. Through appropriate signal processing, the information can be transformed into a one-dimensional velocity map. This has been done quite successfully in the spatial domain, where the specimen is relatively homogeneous. For heterogeneous specimens, the spatial domain technique often fails. A new technique has been developed to reduce the interferograms to a one-dimensional velocity map. This approach operates in the spatial frequency domain and appears to be more reliable for heterogeneous specimens than the spatial domain approach. The theory and implementation of the spatial frequency domain technique (SFDT) are reported, and the two techniques are compared with each other. SFDT utilizes a smaller pixel size than the spatial domain technique. In addition SFDT is faster and more noise tolerant. The extension of the SFDT to produce two-dimensional velocity distribution images is also reported.
\end{abstract}

\section{INTRODUCTION}

$\mathrm{T}$ THE SCANNING LASER acoustic microscope (SLAM) Sonomicroscope $100^{1}$ provides the capability to investigate, quantitatively, ultrasonic propagation properties of specimens on a scale of tens of micrometers. The quantitative assessment of the ultrasonic velocity has the potential of providing valuable details of microscopic elastic properties. There have been three basic measurement schemes described for the ultrasonic velocity determination at a frequency of $100 \mathrm{MHz}$ with SLAM [1]-[3]. All three schemes require SLAM to operate in its interferometry mode in which an interferogram is produced. The photograph in Fig. 1 from the SLAM's TV monitor shows a typical interferogram. The vertical interference lines represent equal phase wavefronts after the sound wave has passed through the specimen. This spatial map of the phase information of the acoustic wave yields the relative sound velocity variations in the specimen. In Fig. 1, a spec-

Manuscript received November 26, 1984; revised February 28, 1985 This work was supported in part by the U.S. National Institutes of Health under Grants CA 36029 and AM 21557 and in part by the Academy of Finland.

K. M. U. Tervola was with the Bioacoustics Research Laboratory, University of Illinois. He is now with the Department of Electrical Engineering University of Oulu, SF 90570 , Oulu 57, Finland.

W. D. O'Brien, Jr., is with the Bioacoustics Research Laboratory, Department of Electrical and Computer Engineering, University of Illinois, 1406 W. Green St., Urbana, IL 61801, USA.

'Sonomicroscope 100 is a registered trademark of Sonoscan, Inc., Bensenville, IL 60106, USA

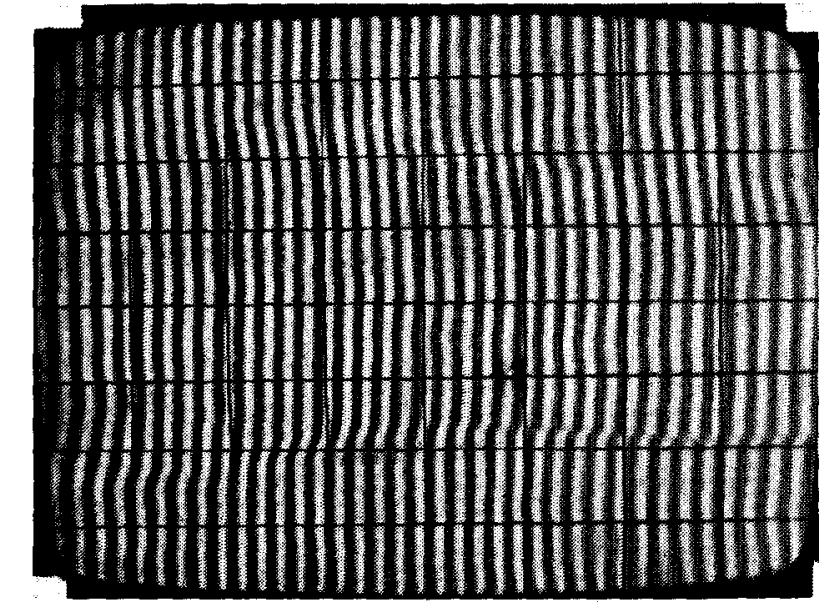

Fig. 1. Interferogram photograph from the SLAM monitor. Specimen is rat liver, and it is positioned horizontally in the middle of the screen. Reference medium (upper and lower parts) in saline. The $8 \times 8$ grid on the monitor facilitates specimen orientation. Field of view is $3 \mathrm{~mm}$ horizontally by $2 \mathrm{~mm}$ vertically.

imen with a velocity greater than that of the surrounding reference medium (saline) has been positioned horizontally across the center of the interferogram. Note that the interference lines shift to the right as they pass from the reference medium, which is above and below the specimen, into the specimen. The field of view is about $3 \mathrm{~mm}$ horizontally by $2 \mathrm{~mm}$ vertically, and there are approximately 39 interference lines, which are spaced at intervals of about 85 micrometers.

With one of the velocity determination schemes, the spatial domain technique (SD'T), the specimen's velocity is determined from the expression [1]

$$
\begin{aligned}
C_{x}= & \left(C_{0} / \sin \theta_{0}\right) \sin \left[\operatorname { a r c t a n } \left(\left(1 / \tan \theta_{0}\right)\right.\right. \\
& \left.\left.-\left(N \lambda_{0} / T \sin \theta_{0}\right)\right)^{-1}\right]
\end{aligned}
$$

where $C_{0}$ is the velocity in the medium surrounding the specimen; $\theta_{0}$ is the angle from the normal of the acoustic beam in the reference medium; $N$ is the normalized fringe shift; $T$ is the specimen thickness; and $\lambda_{0}$ is the wavelength of sound in the surrounding medium. The normalized fringe shift $N$ is determined by dividing the horizontal distance that the fringe has shifted from the reference medium by the horizontal distance between the interfer- 

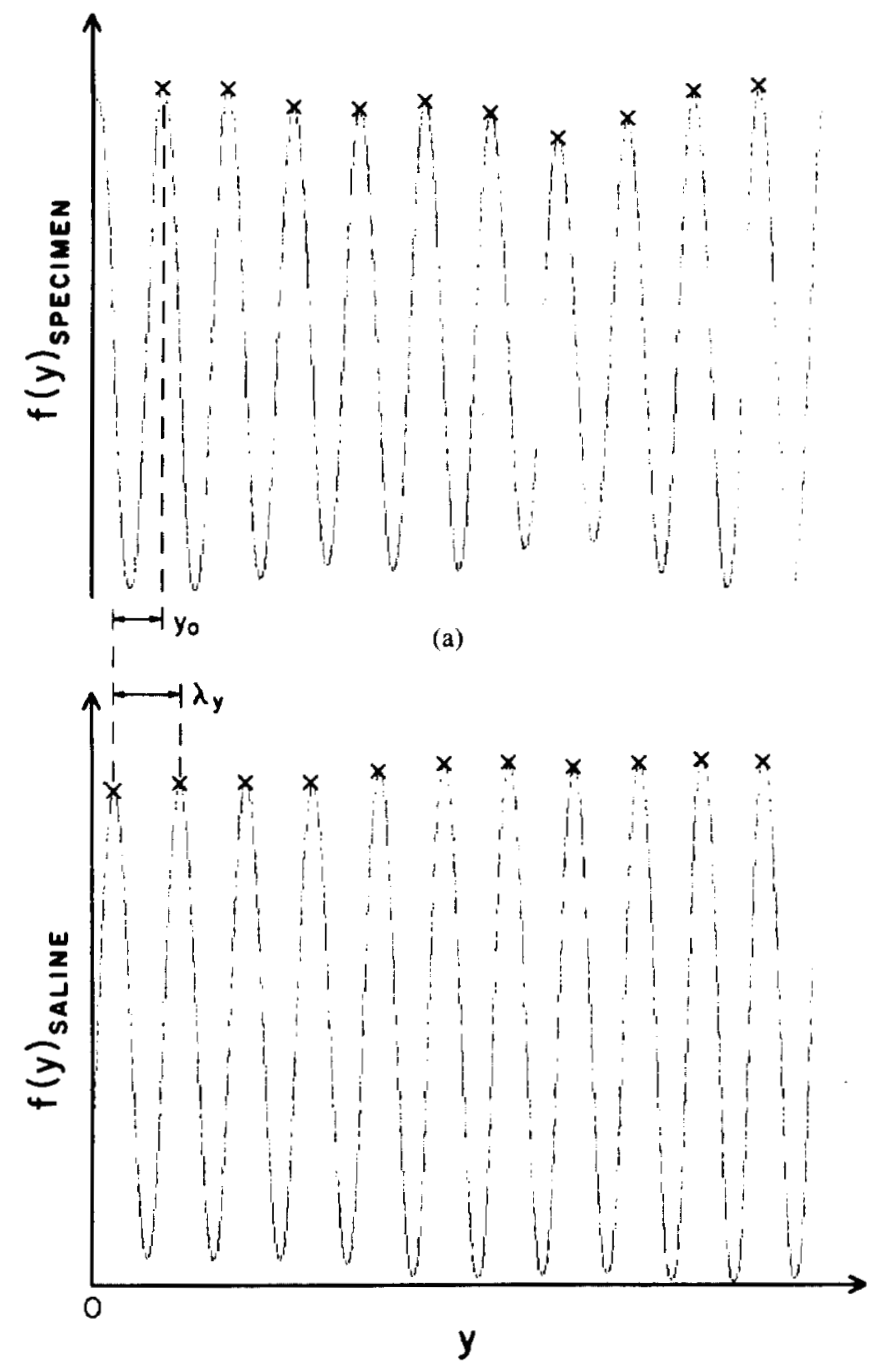

(b)

Fig. 2. The left-most portion of two computer-processed raster lines of the interferogram shown in Fig.1. The raster line through rat liver (a) is shifted to the right with respect to that through saline (b) by the amount of the normalized fringe shift $N=y_{\mathrm{g}} / \lambda y$

ence lines. The numerator of $N$ is assessed at a location in the image where the specimen thickness $T$ is known. A shift to the right for a constant $T$ yields a positive value of $N$ and hence $C_{x}>C_{0}$. The early application of this technique was accomplished by directly measuring these distances from either the SLAM's monitor or from a photographic record of the monitor (like Fig. 1), thus in general yielding a single value for ultrasonic velocity for each interference line [1], [4].

More recently, the determination of velocity by the spatial domain technique has been automated to allow for computer enhancement of the interferogram [5]-[7]. This enhancement process is demonstrated in Fig. 2, which shows the digitized left most portion of two raster line signals of the Fig. 1 interferogram. The lower raster signal $f(y)_{\text {saline }}$ is from the reference medium, in this case saline, and the upper raster signal $f(y)_{\text {specimen }}$ is from the specimen. Fig. 3 schematically depicts this process at the microscope stage where the two $f(y)$ signals of Fig. 2 are

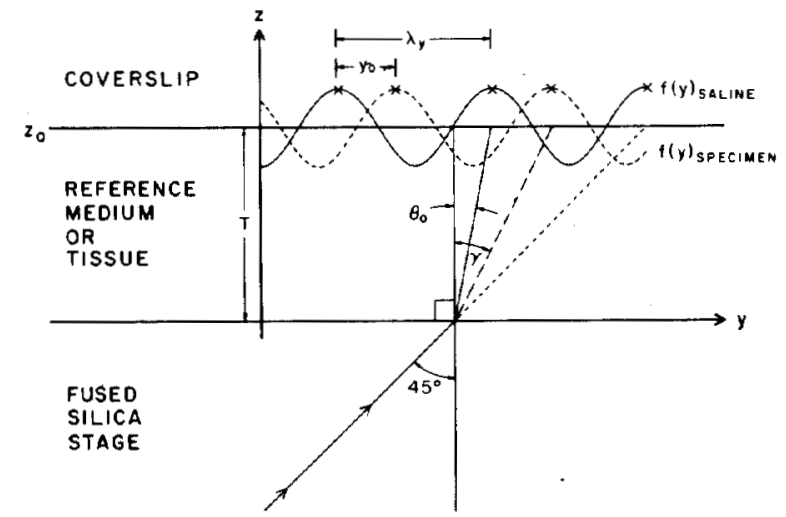

Fig. 3. Schematic illustration on the microscope stage of the two $f(y)$ signals described in Fig. 2. The angles $\theta$ and $\gamma$ represent the refracted wave from the fused silica stage through the reference medium and specimen, respectively.

shown at the lower surface of the coverslip. In principal, each of these signals represents the constructive and destructive interference of two acoustic signals, one that has passed through either the specimen or reference medium and the other that has passed through the reference medium. The sound source of the $100-\mathrm{MHz}$ continuous plane wave is incident at the fused silica-specimen surface at an angle of $45^{\circ}$. The refracted angle of sound into the reference medium is $\theta_{0}$, whereas that into the specimen is $\gamma$. When saline is used as the reference medium $\left(C_{0}=1507\right.$ $\mathrm{m} / \mathrm{s}), \theta_{0}=10.29^{\circ}$. A correlator receiver is used to compare the signal of the raster line with a stored replica of the ideal cross section of an interference line maxima [8]. At the locations of maximum correlation, the center of each of the interference lines for that raster line is noted. In Fig. 2, this is denoted by the $X$ 's on the $f(y)$ signals. This enhancement process proceeds for all 482 raster lines, for which there are about 39 interference line positions noted per raster line. A single interference line then consists of 482 data points and can yield up to 382 individual normalized fringe shift data points $\left(N=y_{0} / \lambda_{y}\right.$ according to Figs. 2 and 3 notation). The specimen is prepared on the microscope stage such that the top and bottom 50 data points of each interference line lie within the reference medium. Provided that the specimen's preparation does not result in an interference line discontinuity at the specimen-reference medium edge, a line-following and smoothing algorithm can be implemented. Then, for each interference line, up to 382 velocity data points are determined. From this velocity distribution, the mean, median, standard deviation, and other statistical parameters can be calculated. This is a considerable improvement (both in terms of time and precision in determining $N$ ) over the manual procedure in that quantitative indices for spatial variation are determined for each interference line. The precision of $N$ is greater; that is, it can be determined to within 0.03 of a fringe as compared to 0.1 manually. The precision is mainly limited because of the quantization error; that is, there are 37 samples taken over each wavelength.

In terms of specimen preparation, the edges are usually 
tapered in order to have continuous interference lines. This is the case for the specimen of Fig. 1, and this is necessary in order to avoid any ambiguity in terms of being able to follow the interference lines continuously. When the thickness at the specimen edge changes abruptly for a specimen that has a different velocity from that of the reference medium, the interference lines likewise shift abruptly at that edge. This produces an ambiguity in terms of which interference line is continuous with that in the reference medium. An excellent description of this problem has been published elsewhere [3]. When this fringe shift ambiguity exists, it is not possible to use the above procedure to determine velocity because the interference lines cannot be followed continuously across the edge and the normalized fringe shift cannot be determined. Two other velocity determination schemes have been devised which address this problem [2], [3]. One involves the preparation of the specimen, usually a hard material, which is cut as a wedge; that is, the specimen has a tapered thickness rather than two parallel surfaces [2]. This scheme is called the wedge-angle technique. The specimen's wedge angle is $\alpha$. When the specimen is placed on the microscope stage with the tapered thickness in the vertical direction, interference lines at an angle $\beta$ from the vertical are produced on the monitor. The specimen velocity, assumed to be homogeneous, is determined from

$$
C_{x}=\left(C_{0} / \sin \theta_{0}\right) \sin \left[\cot ^{-1}\left(\cot \theta_{0}-\cot \alpha \cot \beta\right)\right]
$$

where $\theta_{0}$ has the same meaning as in (1).

The other velocity determination scheme, called spectral interferometry [3], also addressed the fringe ambiguity problem. Here, the displacements of the interference lines are measured on each side of the specimenreference medium edge as a function of frequency. The SLAM operates over a continuous tuneable frequency range from about 95 to $105 \mathrm{MHz}$. In practice, the frequency is adjusted so that the interference lines appears to be vertically continuous at the edge without any shift. Then, when the frequency is changed from $f_{1}$ to $f_{2}$, the interference lines move horizontally in the same direction but with different displacements. The second frequency $f_{2}$ is noted when the inferference lines, again, appear to be vertically continous at the edge without any shift. This represents a differential fringe shift of one. This process is continued to determine the slope of the differential fringe shift versus frequency $d N / d f$ from which $\theta_{x}$, the angle from the normal of the acoustic beam in the specimen, can be determined by [3].

$$
\theta_{x}=\cot ^{-1}\left[\cot \theta_{0}-\left(C_{0} / T \sin \theta_{0},(d N / d f)\right] .\right.
$$

The sound velocity in the specimen can then be determined from Snell's law.

Of these three schemes, the spatial domain technique has found the greatest application for determining the ultrasonic velocity in biological tissues because it is relatively easy to prepare the tissue specimen, such that the interference lines are continuous across the specimen-ref- erence medium boundary. But, a major requirement is that the tissue must be relatively homogeneous because otherwise the fringe shift ambiguity problem exists within the heterogeneous specimens. Then the spatial domain technique often fails because of the lack of continuity of individual interference lines within the area of the specimen. The wedge angle technique is too restrictive for biological specimens because of its requirement for specimen preparation. If the specimen can be prepared, the technique is mainly applicable to relatively homogeneous specimens. The spectral interferometry technique also requires relatively homogeneous specimens, although it is the superior procedure for assessing the fringe ambiguity problem at the specimen-reference medium edge.

None of these three schemes is well suited for assessing the velocity distribution of specimens that are heterogeneous. A new scheme has been devised for the determination of the normalized fringe shift $N$ in heterogeneous specimens. This technique is called the spatial frequency domain technique (SFDT). This paper describes the theoretical background of the SFDT and also gives the computer simulation and experimental assessment results. In addition, the two dimensional SFDT is introduced.

\section{Spatial Frequency Domain Technique}

\section{A. Theoretical Background}

Fig. 4 depicts a single raster signal $f(y)$, which will be used to illustrate the basic approach to determine the normalized fringe shift $N$. The $y$-axis can be thought of as the specimen-coverslip boundary (the $z=z_{0}$ plane in Fig. 3 ). The wavefronts are incident upon the $x y$ plane at an angle $\alpha$, which is either $90^{\circ}-\theta_{0}$ for $f(y)_{\text {saline }}$ or $90^{\circ}-\gamma$ for $f(y)_{\text {specimen }}$ as shown in Fig. 3. The medium wavelength is $\lambda_{\alpha}$, and the wavelength of $f(y)$ is $\lambda_{y}=\lambda_{\alpha} / \cos \alpha$. For example, for the saline reference medium $\left(C_{0}=1507 \mathrm{~m} / \mathrm{s}\right.$ and $\left.\theta_{0}=10.29^{\circ}\right), \alpha=79.71^{\circ}, \lambda_{\alpha}=\lambda_{0}=15.07 \mu \mathrm{m}$, and $\lambda_{y}=84.5 \mu \mathrm{m}$, which is the interference line spacing. This spacing is the same for both the saline and the specimen. The Fourier transform of $f(y), F\{\xi\}$, is its plane wave spectrum (or spatial frequency spectrum). The Fourier transform $F\{\xi\}$ is symmetric because $f(y)$ is a real-valued signal. The spatial frequency $\xi_{0}$, where the power spectrum reaches its maximum, can be derived from

$$
\xi_{0}=\frac{1}{\lambda_{y}}=\frac{\cos \alpha}{\lambda_{\alpha}}
$$

From the Fourier transform pair

$$
f(y) \Leftrightarrow F\{\xi\}
$$

the spatially shifted signal is represented by

$$
f\left(y-y_{0}\right) \Leftrightarrow e^{-j 2 \pi \xi y_{0}} F\{\xi\}
$$

which is the origin shift theorem [9]. The power spectrum of $f\left(y-y_{0}\right)$ is the same for all $y_{0}$, but the phase information of the different spectral components $\phi(\xi)$ has been changed by an amount of

$$
\Delta \phi(\xi)=\phi_{2}(\xi)-\phi_{1}(\xi)=-2 \pi \xi y_{0}
$$




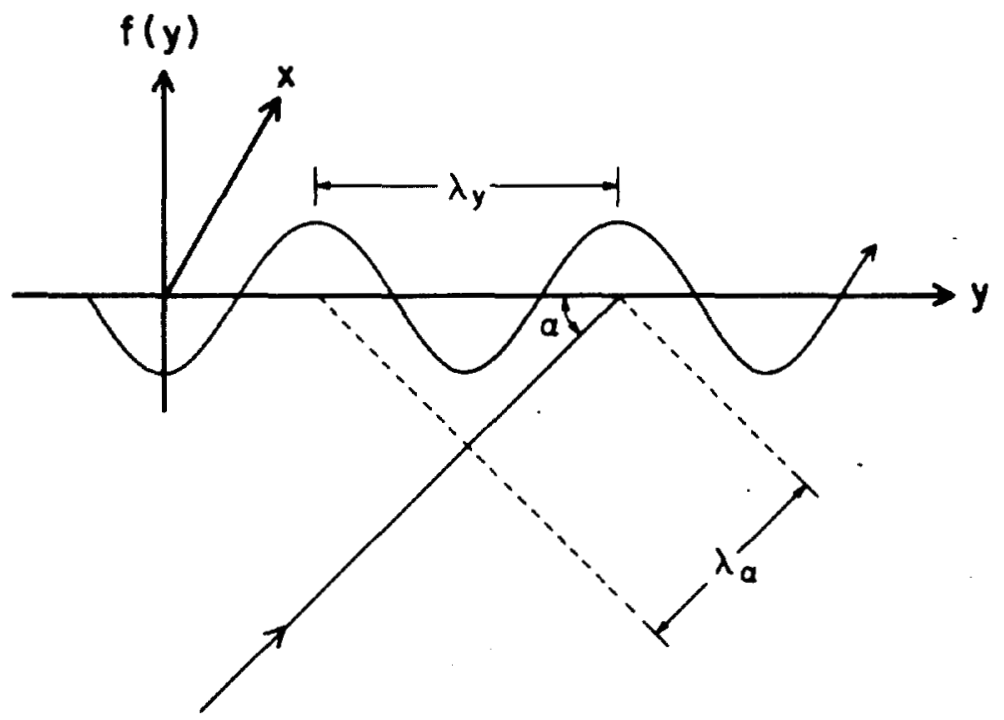

Fig. 4. Illustration of the normalized fringe shift determined with the spatial frequency domain technique.

for all $\xi$ and $y_{0}$. For this application $\phi_{2}(\xi)$ and $\phi_{1}(\xi)$ are the phases of $f(y)_{\text {specimen }}$ and $f(y)_{\text {reference, }}$, respectively. For a fixed spatial frequency component, for example $-\xi_{0}$, the phase shift is

$$
\Delta \phi\left(-\xi_{0}\right)=2 \pi \xi_{0} y_{0}
$$

and from (4)

$$
\Delta \phi\left(-\xi_{0}\right)=2 \pi y_{0} / \lambda_{y}
$$

This shows that $\phi\left(-\xi_{0}\right)$ is directly related to the normalized fringe shift

$$
N=\frac{\Delta \phi\left(-\xi_{0}\right)}{2 \pi} .
$$

The main problems that arise from the implementation of this theory are 1) the fringe-shift ambiguity due to possible abrupt speed changes within the specimen (constant thickness assumed), 2) the modulo $2 \pi$ uncertainty due to the cyclic nature of the Fourier transform, 3) the finite length of the digitized $f(y)$ signal, and 4) noise. The fringe-shift ambiguity and the modulo $2 \pi$ uncertainty problem can be addressed by preparing the specimen properly and by using a computer algorithm to unwrap the phase information, respectively. The specimen is prepared so as to greatly minimize situations at the specimen edge where the absolute value of the normalized fringe shift between two adjacent raster line signals could be greater than 0.5. A computer algorithm addresses the modulo $2 \pi$ uncertainty and is discussed subsequently in some detail. Section II-B evaluates, with computer simulations the effect of the finite detection range of $f(y)$ and that of noise.

\section{B. Simulation Program}

A simulation program has been designed to evaluate the properties of the spatial frequency domain technique for a finite length digitized raster signal and for noise. The program consists of a subprogram, which creates a two-di-

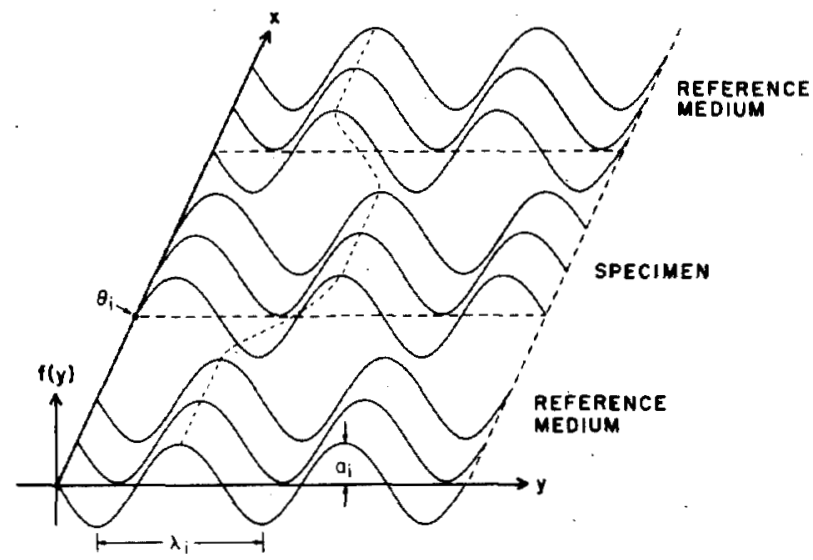

Fig. 5. Illustration of the creation of a simulated interferogram, which is then used to verify the spatial frequency domain technique under ideal conditions.

mensional interferogram and a fast Fourier transform FFT algorithm which analyzes the fringe shift information.

Fig. 5 perspectively illustrates the simulated interferogram of ideal interferogram raster signals without noise. Three subfields are created so that the total field resembles a typical reference medium-specimen-reference medium interferogram like that of Fig. 1. The raster lines of the noise-free subfields in reference medium $i$ are represented by

$$
T_{i}(m, n)=a_{i} \cos \left(2 \pi r / \lambda_{i}+\theta_{i}\right)
$$

where parameters $m$ and $n$ define the pixel coordinates (origin is situated at $m=1, n=1$ ), $a_{i}$ is the amplitude, $\lambda_{i}$ is the wavelength.(about $75 \mu \mathrm{m}$ ), $\theta_{i}$ is the phase when $m=1$, and $r$ is the distance from the $x$-axis and is equal to $m-1$. The dotted line in Fig. 5 depicts a typically shaped interference line.

The model used to study the effect of noise for the measured signal is

$$
g_{i}(y)=f_{i}(y)+n_{i}
$$


and for the simulated signal

$$
T_{i g}(m, n)=T_{i}(m, n)+n_{i}
$$

where $n_{i}$ is the noise factor. The subscript $i$ defines the medium (one for the reference medium and two for the specimen). The probability distribution functions for both media are assumed to be ergodic.

To determine the noise factor $n_{i}$ the probability distribution function of the signal $g_{i}(y)$ was experimentally determined at two fixed points in the interferogram, one in the reference medium (saline) and the other in a specimen. For the simulation, the specimen was a $150-\mu \mathrm{m}$-thick dog skin section, a relatively lossy and heterogeneous specimen. Thus the two distributions were distinct and different in order to exploit the capability of the SFDT and the comparison with the SDT. The signal-to-noise ratios were approximately $24 \mathrm{~dB}$ and $18 \mathrm{~dB}$ for saline and skin, respectively. In both cases, the noise was Gaussian distributed [10]. In the simulations, noise was added into the ideal sinusoids so that the probability distributions were equal to those measured in saline or in the dog skin.

For the determination of the fringe shift $N$ from (10) an FFT algorithm was used. The periodicity (the wavelength) of the actual measurements is about 37 samples. The analysis cannot detect the proper spatial frequency with the window length less than the wavelength. However, the approach appears to work if the spatial frequency $-\xi_{0}$ is constant throughout the whole analysis. In this situation, the phases of the first negative spectral components are used. Otherwise, the theoretical maximum negative spectral components are used. The analysis uses the nearest neighbor approximation and does not use any kind of interpolation methods.

Two simulation studies were performed with $T_{i}(m, n)$, one without noise and the other with noise. The finite window length, the variable studied, ranged from four to 1024 data points. In both studies the fringe shift was assumed to be 0.6 , which is a typical value for actual measurements. All interferograms were digitized to eight bits, the same as in the measurement system. It should be noted that a priori knowledge of the fringe shift has been used in the error analysis because of the $2 \pi$ ambiguity. However, as will be shown later, with an appropriate specimen shape and with the unwrapping of phase, it is still possible to analyze the fringe shift.

The results of both simulation studies are given in Table I. The length of the window varied in powers of two from four to 1024 samples, where a complete raster line contains 1514 data points. The minimum length was four samples because with two samples only two zero-frequency components are left, and their phase is constant for all fringe shifts. The error in the fringe shift is in the range up to 2.7 percent for window lengths of at least 32 samples. For shorter window lengths, the error greatly increases. The added noise to the ideal sinusoids in general increases the error, as should be expected. However, it is interesting to note that the error appears to go through a

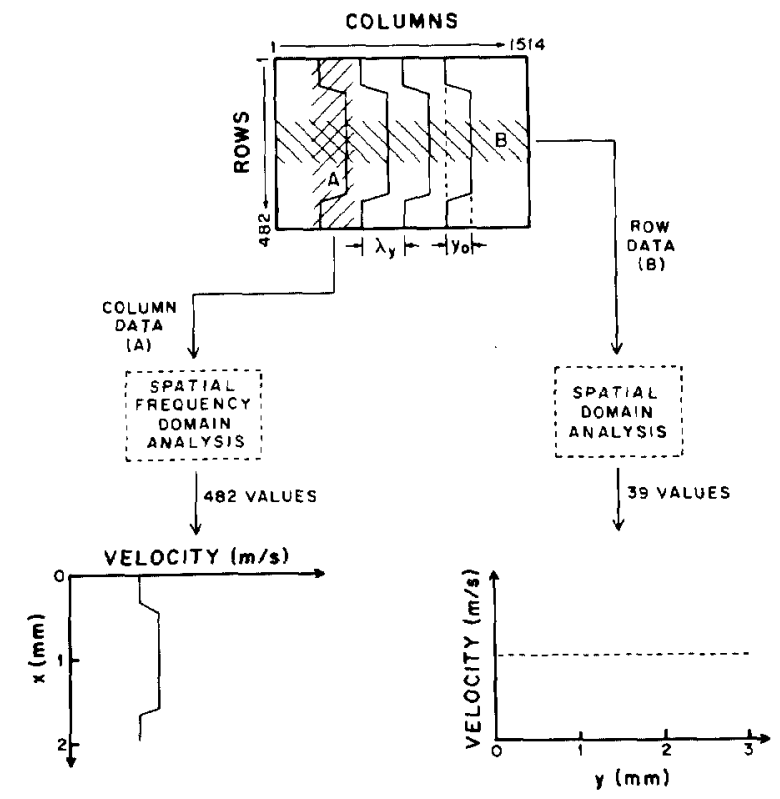

Fig. 6. Schematic representation of the spatial domain and the spatial frequency domain techniques.

TABLE I

Evaluation of ERror in DEtermining the Fringe SHIFT AS a FUnCTION OF THE Window LENGTH

\begin{tabular}{ccc}
\hline & \multicolumn{2}{c}{ Error (percent) } \\
\cline { 2 - 3 } Window Length & Without Noise & With Noise \\
\hline 1024 & 0.0 & 0.5 \\
512 & 0.0 & -0.8 \\
256 & 0.0 & -0.8 \\
128 & -0.8 & -2.7 \\
64 & -0.8 & -2.7 \\
32 & 1.3 & -1.6 \\
16 & -4.0 & -2.9 \\
8 & 14.1 & 14.9 \\
4 & 12.2 & 46.7 \\
\hline
\end{tabular}

'Either ideal sinusoids or sinusoids, where noise was added were used. The normalized fringe shift introduced into the simulation was 0.6 .

minimum for a window length approximately the same size as that of the wavelength which is what would be expected from discrete Fourier transform theory. The data give support to the idea that the analysis is functioning with window lengths of at least 32 samples.

\section{Experimental Evaluation}

A schematic representation of the SFDT is given in Fig. 6. To calculate the fringe shift $N$ in the spatial frequency domain, the FFT algorithm utilizes a vertical set of finite length raster lines $f(y)$. For example, if $f(y)$ is chosen to be 32 data points wide, then the data set is an array 32 data points horizontally by 482 lines vertically. All 482 finite length $f(y)$ 's are positioned the same distance from the left edge of the interferogram (shaded area $A$ ).

The problem described previously as to the cyclic nature of the Fourier transform arises because there exists the modulo $2 \pi$ uncertainty of the phase. The interference lines 


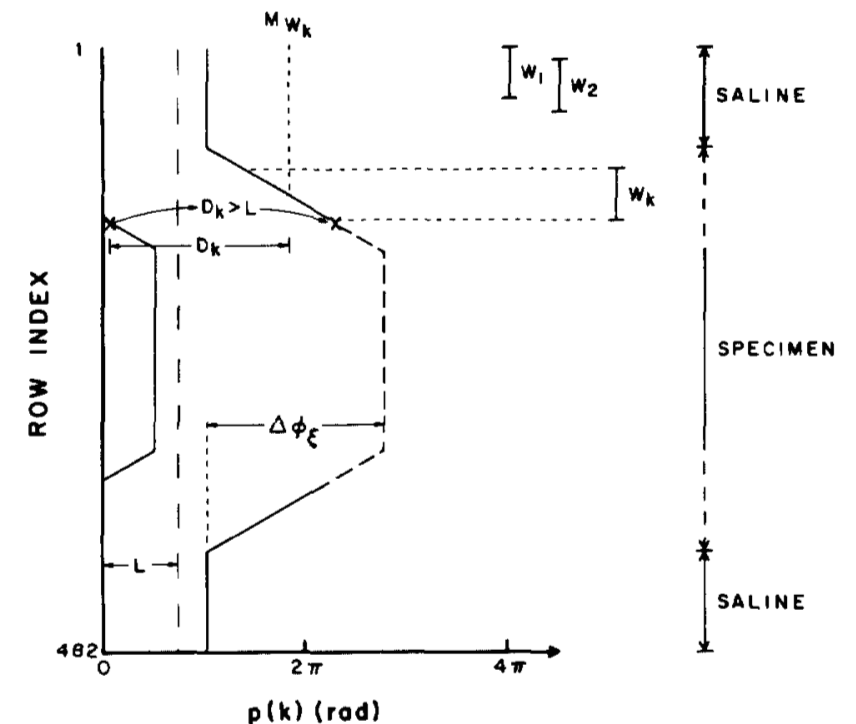

Fig. 7. Illustration of the modulo $2 \pi$ uncertainty problem discovered in the phase information and the parameters of the algorithm designed to solve this problem.

represent constant phase of the acoustic wave that is incident upon the lower surface of the coverslip. However, the discrete phase value (noted in Fig. 7 as $p(k)$ ) derived from the FFT algorithm for the finite length raster line signal can change from one raster line to the next within the data set. These values of $p(k)$, where $k$ represents the finite $f(y)$ raster line row index, throughout the data set can vary by more than $2 \pi$, although the FFT algorithm will yield a phase value within the range $\pm \pi$. This problem is dealt with by using a running-mean algorithm, which assumes that $p(k)$ is continuous at the beginning from one raster line to the next. Here, the beginning is the upper portion of the interferogram which, because of specimen preparation on the microscope stage, is the homogeneous reference medium, saline. A mean value of $p(k), M_{W_{k}}$, is determined from several adjacent rows and these rows are noted in Fig. 7 as ranges $W_{1}, W_{2}, \cdots, W_{k}$, because this window runs from the top to bottom of the data set. The difference $\left(D_{k}\right)$ between $M_{W_{k}}$ and the next $p(k)$ value is determined. This difference is compared to the decision level $L$. Through this process the continuity of the interference line is obtained. Once this process is completed, the value of $\Delta \phi(\xi)$, as shown in Fig. 7, is obtained for each raster line value within the specimen. From (10), the normalized fringe shift is calculated and hence the speed of sound for a known specimen thickness. If the number of samples for the FFT algorithm is at least equal to 32 , then the data width is nearly an integer multiple of the wavelength $\lambda_{y}$, which assures the minimum amount of leakage in the Fourier analysis with the smallest pixel size.

Fig. 8 shows an example of a velocity profile for a 370 $\mu$ m-thick normal rat liver specimen. The window length was 32 samples and 482 speed values were calculated. The speed profile illustrates the shape of the specimen, which was tapered at the edges so that the phase $p(k)$, shown schematically in Fig. 7, did not change too rapidly. The

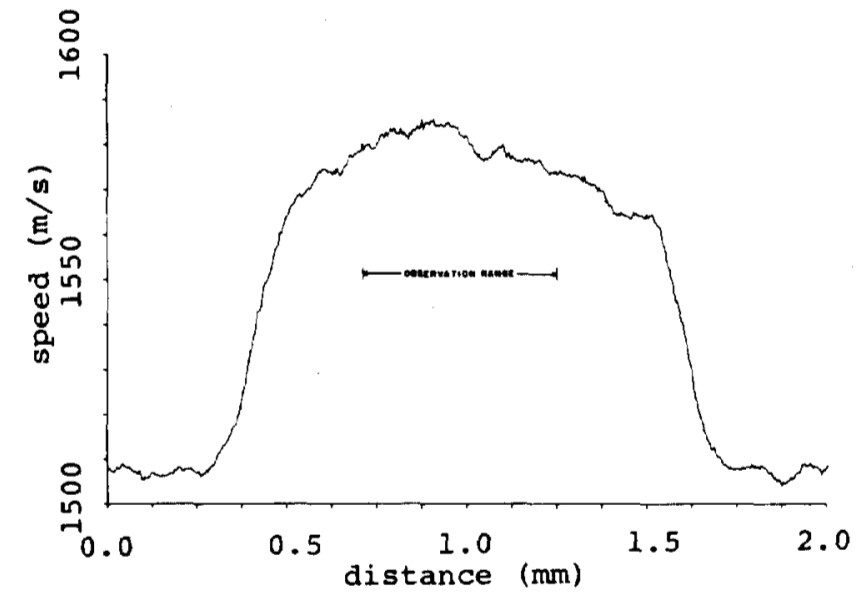

Fig. 8. Result of the spatial frequency domain analysis for a $370-\mu \mathrm{m}$-thick rat liver specimen. The average velocity value taken in the observation range should be compared to the value labeled by label 2 in Fig. 12 .

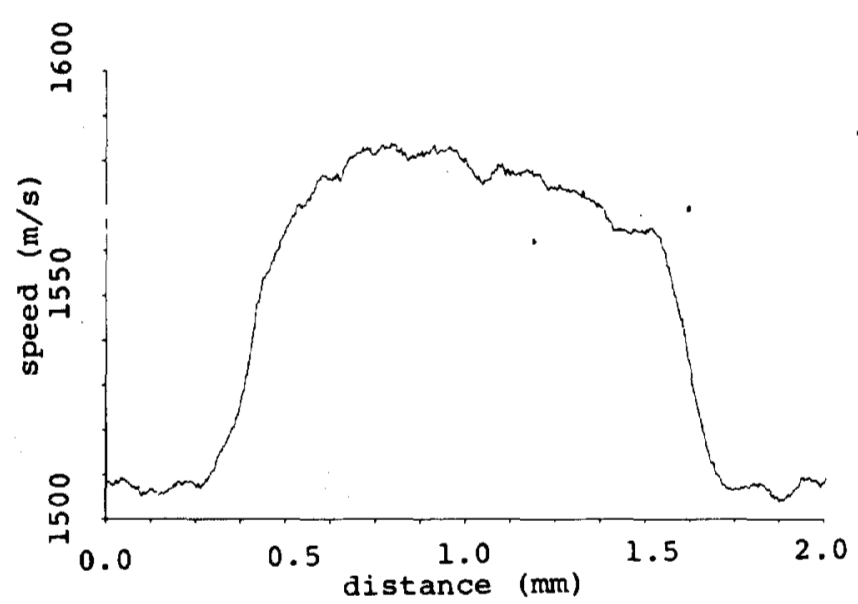

Fig. 9. Result of the spatial frequency domain analysis of the same interferogram as used in Fig. 8, where the window has been shifted 32 data points to the right. Now the average velocity in the observation range should be compared to the value pointed out by label 3 in Fig. 12 .

notation observation range in Fig. 8 represents the area of the specimen of known and constant thickness. Along this $64-\mu \mathrm{m}$-wide vertical strip through the specimen a mean value of $1580.0 \mathrm{~m} / \mathrm{s}$, and a standard deviation of $3.5 \mathrm{~m} / \mathrm{s}$ has been determined.

The SFDT analysis was repeated with three additional adjacent, 32 sample windows of the same $370-\mu \mathrm{m}$ rat liver specimen. The results are given in Table II. For the second run the velocity profile is shown in Fig. 9. When comparing the results of the four runs with each other, it is noted that the velocity profiles and the average values in the observation range are essentially the same. This is expected because the liver specimen is relatively homogeneous.

The velocity values listed in Table II for the spatial frequency domain analysis are achieved by using a window length of 32 samples. The analysis was repeated for window lengths of eight and four samples from the same relatively homogeneous specimen. The window with 32 data samples (noted as the third run in Table II) is then divided into four adjacent subwindows, each eight samples long. 


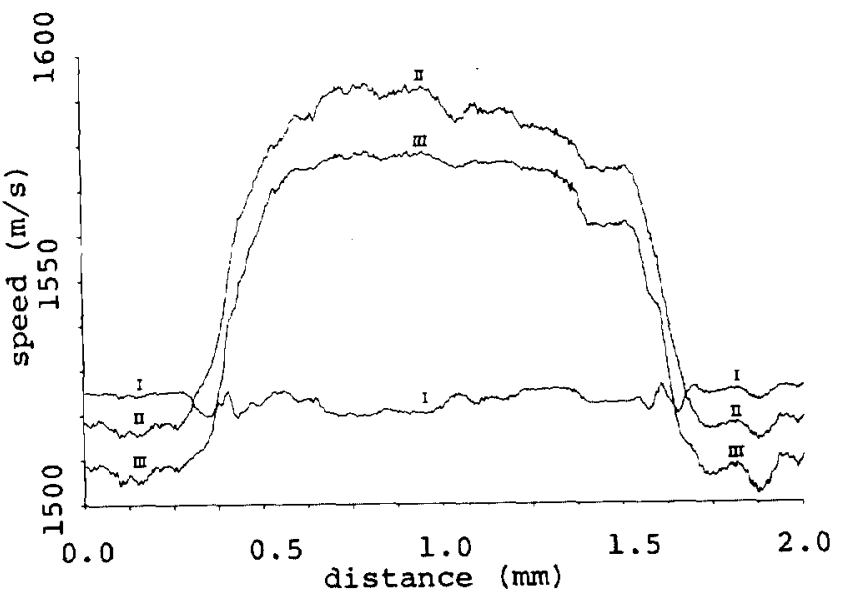

Fig. 10. Velocity profiles along the 370- $\mu$ m-thick rat liver specimen. Curve II is the same as in Fig. 9, but it has been shifted upward $10 \mathrm{~m} / \mathrm{s}$. Curve III is plotted for the average values of four velocity sets, which were the results of splitting the window used in Fig. 9 into four subwindows. Curve I is the differences between curves II and III. The zero value for curve I is at the offset level of $1525 \mathrm{~m} / \mathrm{s}$.

TABLE II

Average Speed Values Given by the Two TECHNIQUES

\begin{tabular}{ccc}
\hline & \multicolumn{2}{c}{ Average Speed of Sound } \\
\cline { 2 - 3 } $\begin{array}{c}\text { Run } \\
\text { Number }\end{array}$ & $\begin{array}{c}\text { From SFDT } \\
(\mathrm{m} / \mathrm{s})\end{array}$ & $\begin{array}{c}\text { From SDT } \\
(\mathrm{m} / \mathrm{s})\end{array}$ \\
\hline 1 & 1580.2 & 1580.8 \\
2 & 1580.0 & 1579.7 \\
3 & 1579.6 & 1578.3 \\
4 & 1578.3 & 1577.2 \\
F1 & 1577.2 & analysis failed \\
F2 & 1574.1 & analysis failed
\end{tabular}

'The same measurement data are used as input.

TABLE III

RESULTS OF WINDOW LENGTH WITH THE SPATIAL FRREQUENCY DOMAIN TECHNIQUE

\begin{tabular}{ccc}
\hline Window Length & $\begin{array}{c}\text { Average } \\
\text { Speed } \\
(\mathrm{m} / \mathrm{s})\end{array}$ & $\begin{array}{c}\text { Standard } \\
\text { Deviation } \\
\text { of }\end{array}$ \\
$\begin{array}{c}\text { the Difference } \\
\text { (curve I }) \\
(\mathrm{m} / \mathrm{s})\end{array}$ \\
\hline 32 & 1579.6 & 3.6 \\
8 & 1572.7 & \\
8 & 1584.1 & \\
8 & 1573.7 & \\
8 & 1575.7 & \\
4 & 1573.7 & 4.6 \\
4 & 1580.2 & \\
\hline
\end{tabular}

The SFDT analysis is performed for each of these subwindows, and the results are listed in Table III. The velocity profiles of the four subwindows were averaged, and the result is plotted in Fig. 10 (curve III), along with the 32-data-sample velocity profile (curve II). Curve II has been shifted by $10 \mathrm{~m} / \mathrm{s}$, so that the two curves are separated and more easily examined. The difference between

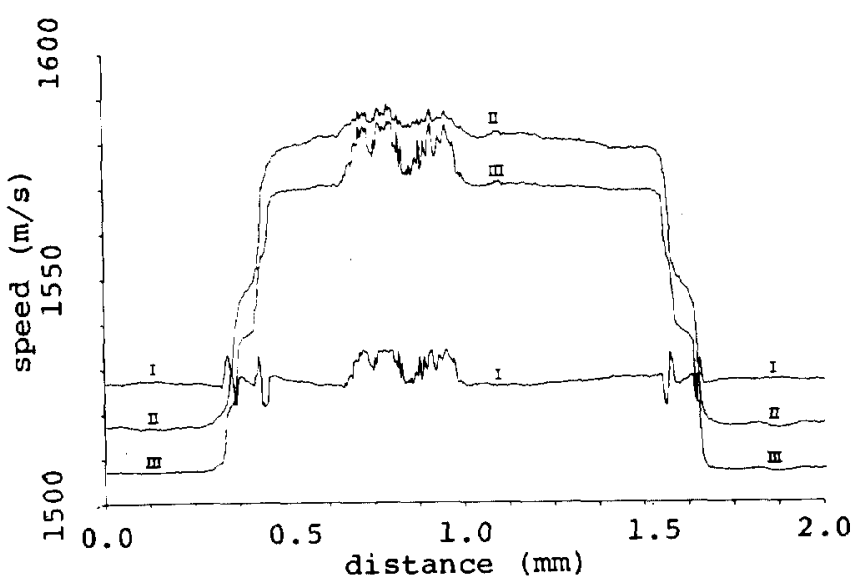

Fig. 11. Velocity profiles along the $370-\mu \mathrm{m}$-thick rat liver specimen. Curve II is the result of an analysis when using the window length of eight samples, and it is shifted $10 \mathrm{~m} / \mathrm{s}$. Curve III is the average of the two runs when the window was split to two subwindows with four samples each Curve I gives the difference between curves II and III. The zero value is at the level of $1525 \mathrm{~m} / \mathrm{s}$. The $+10 \mathrm{~m} / \mathrm{s}$ offset of curve II when drawing curve $I$ is taken into account.

these two curves is shown in Fig. 10 as curve I. The individual average velocity value for each of the window lengths is listed in Table III. The mean value for the four subwindows is $1576.6 \mathrm{~m} / \mathrm{s}$, which is within $3 \mathrm{~m} / \mathrm{s}$ of the mean value for all 32-data-sample analysis. The standard deviation of curve $I$ in Fig. 10 is $3.6 \mathrm{~m} / \mathrm{s}$. The difference of the average velocity for each of the four eight-data-sample subwindows is less than \pm 0.5 percent.

The analysis was repeated using two adjacent lengths of four samples each. One of the previous analyzed windows of eight data samples was subdivided. The results are shown in Fig. 11 (the curves are labeled in the same way as in Fig. 10) and listed in Table III. The mean value for the two four-data-sample analyses is $1575.7 \mathrm{~m} / \mathrm{s}$, which is within $2 \mathrm{~m} / \mathrm{s}$ of the eight-data-sample analysis. The standard deviation of curve I is $4.6 \mathrm{~m} / \mathrm{s}$. These observations support the idea that the spatial frequency domain technique can provide reliable velocity values with a window length of only four samples for relatively homogeneous specimens. Spatially, this represents a pixel size of about one ninth of a wavelength. In this case the pixel size is $4 \times 8 \mu \mathrm{m}$.

The theory and implementation of the spatial frequency domain technique have been presented. Velocity profiles of a rat liver specimen has been demonstrated. To continue the evaluation, the SFDT is now compared to the SDT for the same specimen.

\section{Comparison of the Spatial Frequency Domain and Spatial Domain Techniques}

The spatial domain technique [6], [7] has been the analysis procedure used to evaluate the ultrasonic velocity in homogeneous biological specimens. This is the only available technique to which the newly proposed spatial frequency domain technique can be compared. The basis of the comparison is with rat liver tissue which is relatively homogeneous. An attempt has been made to compare the 
two techniques with a more heterogeneous specimen, but the SDT could not properly analyze the results.

The basic difference between the two techniques is shown schematically in Fig. 6 . The input data set for both is the same digitized interferogram which consists of 1514 columns and 482 rows for a total of 729748 pixels. The pixel size is 2 by $4 \mu \mathrm{m}$ since the field of view is $3 \mathrm{~mm}$ horizontally by $2 \mathrm{~mm}$ vertically, respectively. The shadowed areas (denoted by A and B in Fig. 6) illustrate those portions of the interferogram that are used with the two techniques. The number of velocity values is about 39 with the SDT and 482 with the SFDT. The SDT determines the velocity values horizontally along the specimen at intervals of $85 \mu \mathrm{m}$, and the SFDT determines them vertically across the specimen at intervals of $4 \mu \mathrm{m}$. In order to compare properly the two techniques, the results are evaluated with each other only in the area where the two shaded regions overlap. It would, of course, be possible to increase the number of velocity values with the SDT by changing the phase of the reference signal thereby shifting the interface lines laterally in $y$. This would have to be performed manually and thus has not been evaluated.

For this comparison, a 370- $\mu \mathrm{m}$ thick specimen of fresh rat liver is used [10]. The results from the SFDT from this preparation have been discussed above. The velocity profile is shown in Fig. 8 for a window length of 32 data samples. This figure also notes a region termed "observation range" which represents a specimen area of known and constant thickness and also denotes the area that overlaps with the region of SDT analysis. The average velocity value within this region was $1580.0 \mathrm{~m} / \mathrm{s}$ as determined with the SFDT.

The vertical width of the region used for the SDT was 75 data samples [6], [7]. The results from the implementation of this technique are shown in Fig. 12. The mean velocity data point which overlaps with the SDFT region of Fig. 8 is labeled by the label 2 in Fig. 12. Its value is $1579.9 \mathrm{~m} / \mathrm{s}$, which compares quite favorably with 1580.0 $\mathrm{m} / \mathrm{s}$ from the SFDT. The SFDT and SDT analyses were performed in adjacent areas of this same specimen. In Table II are listed the corresponding values for three other regions which overlap. These are labeled with labels 1,3 , and 4 in Fig. 12 of the SDT. The velocity profile corresponding to the data point labeled 3 in Fig. 12 is shown in Fig. 9 for the SFDT. The SFDT profiles corresponding to label 1 and 4 in Fig. 12 are not shown because they are very similar to those already shown in Figs. 8 and 9 . The results show that the two techniques yield virtually the same average velocity values for this specimen in the same observation area. It is also interesting to observe that for both techniques they show the same trend for decreasing value of the average velocity from areas labeled 1 through 4 in Fig. 12.

The spatial domain technique is subject to failure. These causes have been discussed elsewhere [6], [7]. Basically, the principal reason is specimen heterogeniety, even on a very localized basis. For the liver specimen of Fig. 12, the SDT failed at two positions, which are noted by F1 and

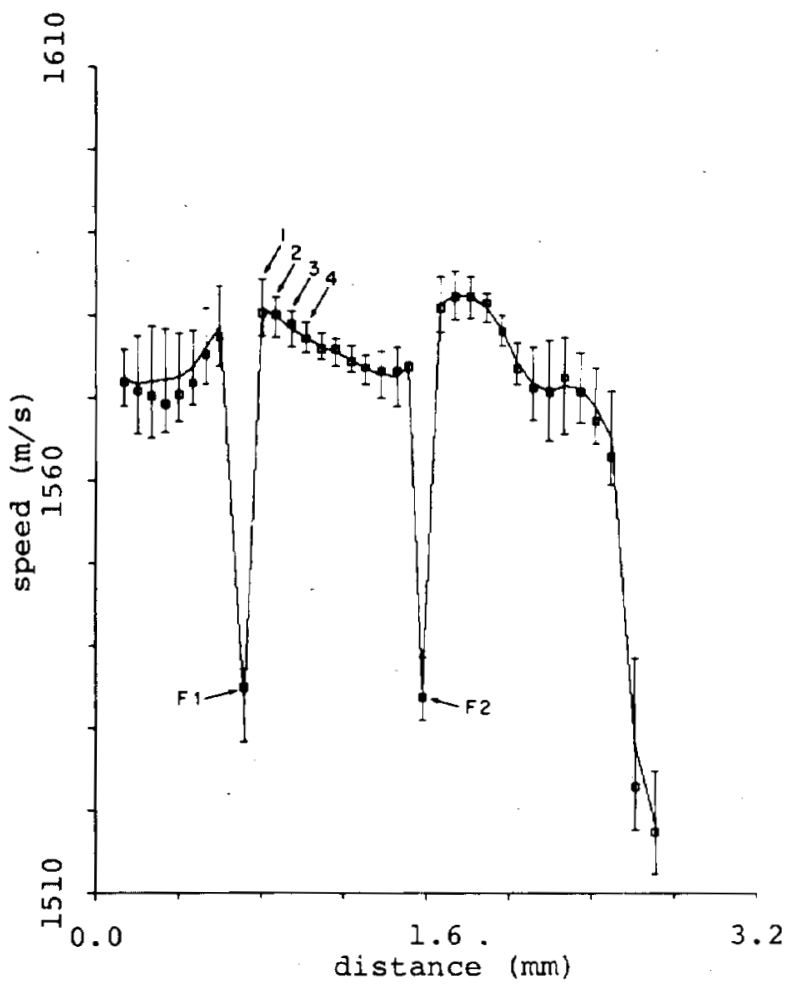

Fig. 12. Result of the spatial domain analysis when using an interferogram taken through a $370-\mu$ m-thick rat liver specimen, where the curve represents average velocity, the squares represent the median velocity, and the vertical bars represent the \pm 1 standard deviation boundaries. The velocity values labeled by labels 2 and 3 should be compared to the average velocity value found in the observation range in profiles given in Figs. 8 and 9, respectively.

F2 because of data discontinuity in the interference lines. An analysis with the SFDT corresponding to the specimen regions of data points labeled by $F 1$ and $F 2$ yielded 1577.2 $\mathrm{m} / \mathrm{s}$ and $1574.1 \mathrm{~m} / \mathrm{s}$, respectively. From the SDT (Fig. 12), if one were to assume that in the regions of $F 1$ and $F 2$ velocity could be obtained by averaging the two adjacent data points on each side of $F 1$ and $F 2$, then the respective values of $1579.7 \mathrm{~m} / \mathrm{s}$ and $1578.0 \mathrm{~m} / \mathrm{s}$ would be obtained. The difference in both cases is less than 0.3 percent, so the conclusion can be drawn that the spatial frequency domain technique is working even in such circumstances where the spatial domain technique fails.

The total time required for the analysis with the spatial frequency domain technique and a window length of 32 samples is about ten minutes. This is about half of the time required for the spatial domain technique. In addition, the number of velocity values obtained from the SFDT and SDT analyses are 482 and 39, respectively, so the spatial frequency domain technique is about 20 times faster than the spatial domain technique for producing a velocity value.

The two velocity techniques have been used to evaluate the velocity distribution of a more heterogeneous tissue specimen-in this case a 75- $\mu \mathrm{m}$-thick section of dog skin that contains a 12-day-old incisional would. The section is cut parallel to the skin surface [11]. Figs. 13 and 14 show the velocity profiles which were determined from the SDT and the SFDT, respectively. The wound area was posi- 


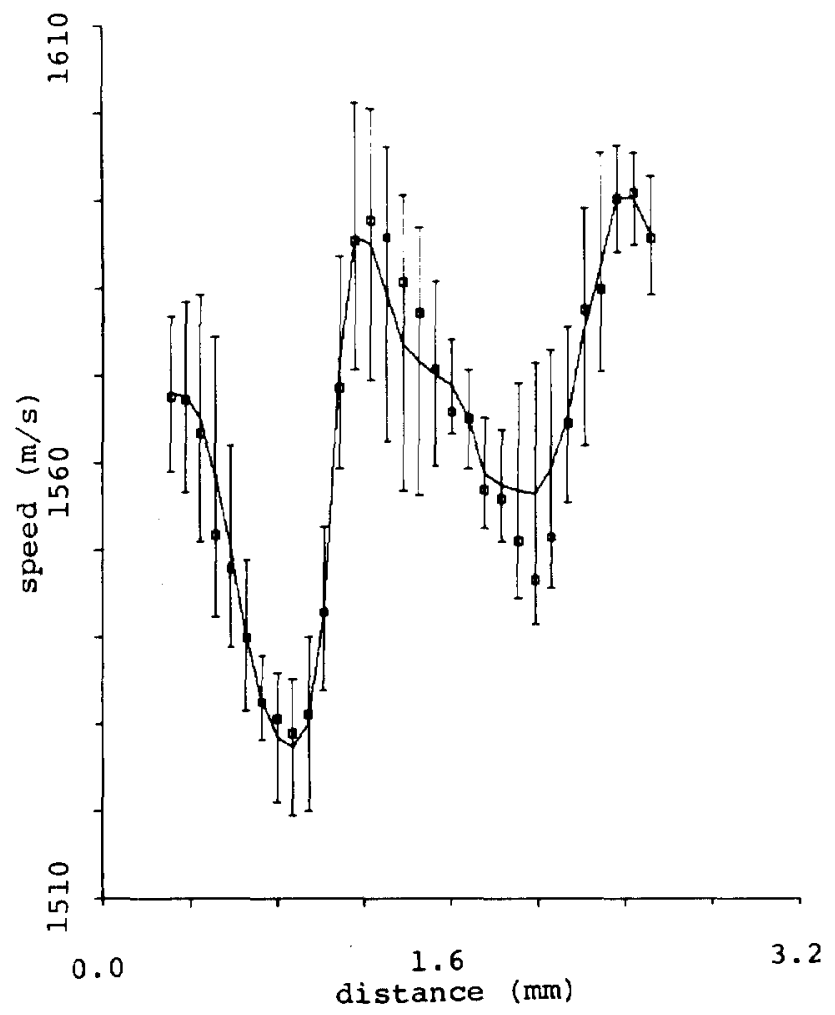

Fig. 13. Velocity profile along a $75-\mu \mathrm{m}$-thick specimen of wounded dog skin with the spatial domain technique. The profile is given in the $y$ direction.

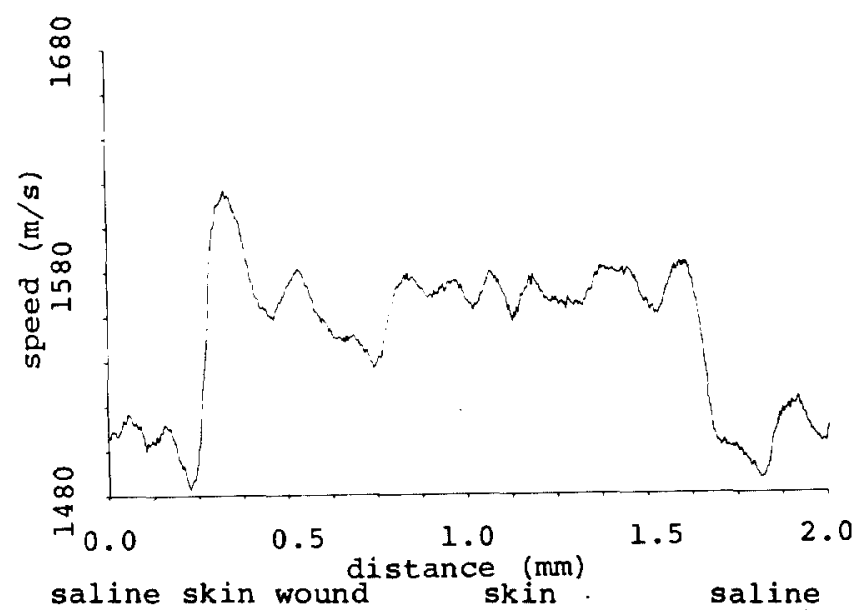

Fig. 14. The output of the spatial frequency domain analysis in the $x$ diretion of the same wounded dog skin specimen as in Fig. 13.

tioned horizontally (along the $y$-axis, see Fig. 6). Above and below the wound margin was skin. The height of the wound area was approximately $300 \mu \mathrm{m}$. Therefore, in applying the SDT analysis procedure (approximately $250 \mu \mathrm{m}$ in width), the region included essentially all of the wound area. However, because there is not a clear indication where the wound margin is, some of the adjacent skin could have been included within the data region. This contributed to the wide variation in the speed as shown in Fig. 13. This is a little clearer where the SFDT analysis procedure (window length of 32 data samples and $64 \mu \mathrm{m}$ wide) was applied in that the values of speed pass through the wound area. Here the approximate regions of saline (above and below the entire specimen), skin and wound are noted in Fig. 14.

Because the two techniques yield the speed profiles along different directions of the specimen a direct comparison is possible only for those areas which overlap. The results are summarized in Table IV for 24 observation areas. Six adjacent regions, each $250 \mu \mathrm{m}$ wide (60 raster lines) were used for the SDT and four adjacent regions, each $64 \mu \mathrm{m}$ wide (32 data samples) were used for the SFDT. For nearly direct comparison between the two techniques, only the interference line, which passed through the overlapping region, was used for the SDT. The overall speed ranged from 1552 to $1597 \mathrm{~m} / \mathrm{s}$. The maximum difference $(\Delta)$ between the two techniques ranged from $-16 \mathrm{~m} / \mathrm{s}$ to $15 \mathrm{~m} / \mathrm{s}$ which is in the range of $\pm 1 \%$ of the nominal values. The difference in part can be attributed to the difference in sample size between the two techniques. However, even for such a heterogeneous sample where the speed extrema was $45 \mathrm{~m} / \mathrm{s}$, in no case did the difference between the two techniques exceed $16 \mathrm{~m} / \mathrm{s}$.

\section{Two-Dimensional SFDT}

Extension of the spatial frequency domain technique to produce a two dimensional speed distribution image is accomplished by sliding the "window" in both the $x$ and $y$ directions. Fig. 15 is a composite of a $75-\mu \mathrm{m}$ section of dog skin which contains a 12-day-old incisional wound. The interferogram Fig. 15(a), acoustic image Fig. 15(b), and optical image Fig. 15 (c) provide a reference. Fig. $15(\mathrm{~d})$ is a gray-scale image that depicts the speed distribution in the specimen. The image consists of 1410 pixels (47 rows $\times 30$ pixels/row); the window length was 32 samples. The gray scale ranges from dark for the lowest speed values to light for the highest, and it is scaled linearly from the minimum velocity value of about 1500 $\mathrm{m} / \mathrm{s}$ (for saline) to about $1650 \mathrm{~m} / \mathrm{s}$ (for hair follicles). The speed in the wound tissue area is about $1540 \mathrm{~m} / \mathrm{s}$, which is lower than that of the skin, $1590 \mathrm{~m} / \mathrm{s}$.

The computational efficiency of the SFDT could be increased by evaluating the Fourier integral numerically only at $-\xi_{0}$. In addition, if the window length would be 37 samples, which is exactly one wavelength, then the errors due to the anomalies of the windowing would be minimized.

\section{Summary}

The principle when performing velocity measurements with the SLAM has been explained. The main part of the measurement is the determination of the fringe shift $N$. Instead of finding the fringe shift with spatial domain calculations, a technique with frequency domain calculations is reported. With simulations, which follow the given theory, and comparing the results of the two techniques with the same measurement data, it has been proven that the spatial frequency domain technique works. In addition, it has some advantages over the spatial domain technique: the pixel size is smaller, the noise tolerance is better, and 
TABLE IV

Average Velocity of Dog SKIN'

\begin{tabular}{|c|c|c|c|c|c|c|c|c|c|c|c|c|}
\hline \multirow{2}{*}{$\begin{array}{l}\text { SDT Analysis Region } \\
(\mu \mathrm{m})(x \text {-direction })\end{array}$} & \multicolumn{12}{|c|}{ SFDT Analysis region ( $\mu \mathrm{m})$ ( $y$-direction) } \\
\hline & SDT & SFDT & $\Delta$ & SDT & SFDT & $\Delta$ & SDT & SFDT & $\Delta$ & SDT & SFDT & $\Delta$ \\
\hline $250-500$ & 1582 & 1587 & 5 & 1581 & 1578 & -3 & 1581 & 1575 & -6 & 1581 & 1588 & 7 \\
\hline $500-750$ & 1556 & 1562 & 6 & 1559 & 1557 & -2 & 1565 & 1552 & -13 & 1582 & 1574 & -8 \\
\hline $750-1000$ & 1570 & 1580 & 10 & 1578 & 1569 & -9 & 1586 & 1570 & -16 & 1592 & 1595 & 3 \\
\hline $1500-1750$ & 1570 & 1580 & 10 & 1568 & 1568 & 0 & 1563 & 1562 & -1 & 1555 & 1570 & 15 \\
\hline
\end{tabular}

'Determined in the same $75-\mu \mathrm{m}$-thick section of dog skin with a 12-day-old incisional wound. Two techniques were employed, the spatial domain technique (SDT) and the spatial frequency domain technique (SFDT). The regions where the two techniques overlapped are directly compared. The difference in speed $(\Delta)$ is indicated.

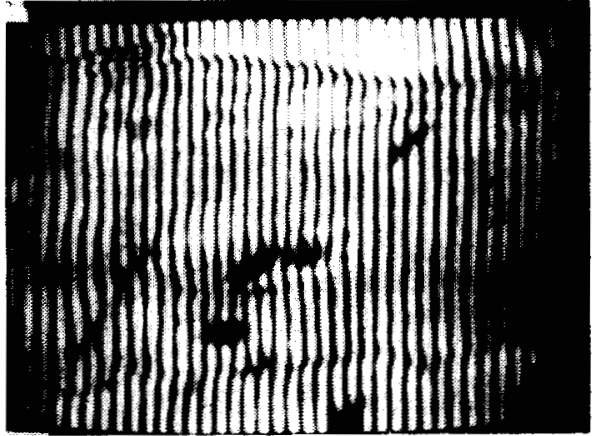

(a)

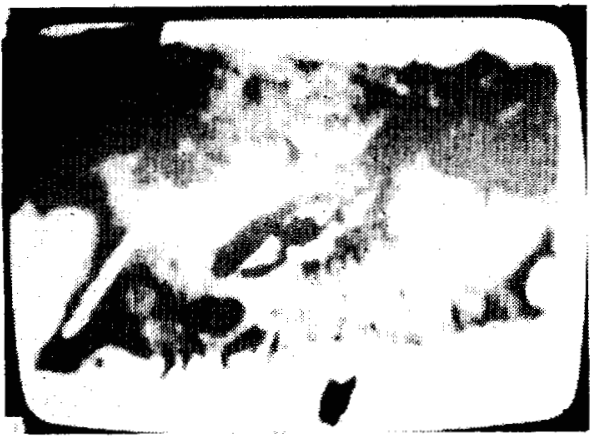

(c)

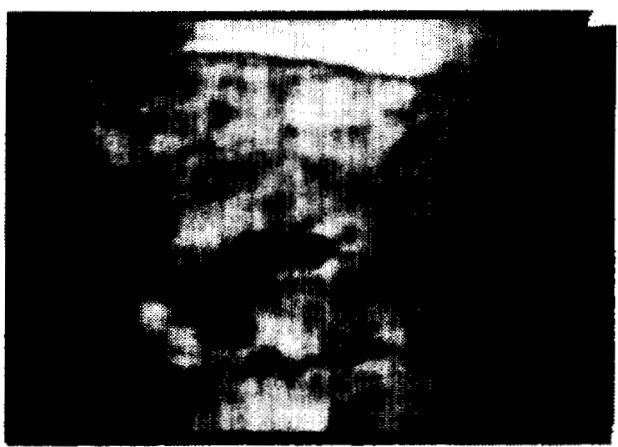

(b)

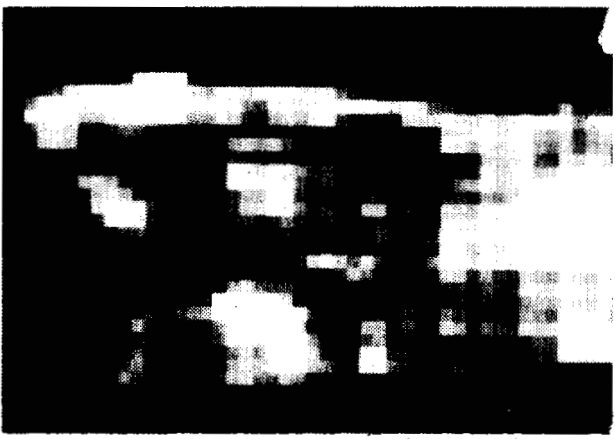

(d)

Fig. 15. Image of a $75-\mu \mathrm{m}$ section of dog skin containing a 12-day-old incisional wound. (a) Interferogram. (b) Acoustic image. (c) Optical image. (d) Two-dimensional velocity distribution. Pixel size of the velocity distribution image is $64 \times 64 \mu \mathrm{m}$, and the light and dark areas correspond to high and low velocity, respectively.

the analysis is faster. The spatial frequency domain technique has also been extended to produce images of twodimensional velocity distributions. The image can be used when studying velocity properties as a function of tissue type in the same heterogeneous specimen, as is the case for the example with wounded dog skin. The first application of the new technique is going to be in the wound healing study, where the change of the ultrasonic velocity properties of the wounded area is studied as a function of time after operation.

\section{REFERENCES}

[1] S. A. Goss and W. D. O'Brien, Jr., "Direct ultrasonic velocity measurement of mammalian collagen threads," J. Acoust. Soc. Amer., vol. 65, pp. 507-511,1979.
[2] M. A. McAvoy and W. D. O'Brien, Jr., "Acoustic velocity using the scanning laser acoustic microscope (SLAM)" in Proc. 1982 IEEE UItrason. Symp., pp. 634-637, cat. no. 82, chap. 1823-4, 1982.

[3] M. G. Oravecz and S. Lees, "Acoustic spectral interferometry: A new method for sonic velocity determination," in Rev. Progress in Quantitative Nondestructive Evaluation, D. O. Thompson and D. E. Chimenti, Eds. New York: Plenum, 1984, vol. 3B, pp. 799-810.

[4] L. A. Frizzell and J. D. Gindorf, "Measurement of ultrasonic velocity in several biological tissues," Ultrasound Med. Biol., vol. 1, pp. 385$387,1981$.

[5] S. G. Foster, "An image digitizing system for scanning laser acoustic microscope," M.S. Thesis, Department of Electrical Engineering, University of Illinois at Urbana-Champaign, IL, 1981.

[6] P. M. Embree, S. G. Foster, G. Bright, and W. D. O'Brien, Jr., "U1trasonic velocity spatial distribution analysis of biological materials with the scanning laser acoustic microscope," in Acoustical lmaging, M. Kaveh, et. al., Eds. New York: Academic, vol. 13, pp. 203-216, 1983.

[7] P. M. Embree, K. M. U. Tervola, S. G. Foster, and W. D. O'Brien, 
Jr. "Spatial distribution of the speed of sound in biological materials with the scanning laser acoustic microscope," IEEE Trans. Sonics Ultrason., vol. 32, no. 2, pp. 341-350, Mar., 1985

[8] H. L. Van Trees, Detection, Estimation and Modulation Theory: Part 1. New York: John Wiley, 1968, p. 277.

[9] J. D. Gaskill, Linear Systems, Fourier Transforms, and Optics. New York: John Wiley, 1978, p. 199.

[10] K. M. U. Tervola, M. A. Grummer, J. W. Erdman, Jr., and W. D. O'Brien, Jr, "Ultrasonic attention and velocity properties in rat liver as a function of fat concentration- $A$ study at $100 \mathrm{MHz}$ using a scanning laser acoustic microscope," J. Acoust. Soc. Amer., vol. 71, pp. $307-313,1985$.
[11] W. D. O'Brien, Jr., J. Olerud, J. M. Reid, and K. K. Shung, "Quantitative acoustical assessment of wound maturation with acoustic microscopy," J. Acoust. Soc. Amer., vol. 69, pp. 575-579, 1981.

Kalervo Tervola for a photograph and biography please see page 265 of the March 1985 issue of this Transactions.

William D. O'Brien, Jr. (S'64-M'71-SM'79). For a photograph and biography please see page 25 of the January 1985 issue of this TRANSACTIONS. 